\title{
Research on Safety Lane Change Warning Method Based on Potential Angle Collision Point
}

\author{
Tongqiang Ding, Xiaorong Li $\mathbb{D}^{D}$, Lili Zheng $\mathbb{D}^{D}$, and Zhiguo Hao \\ JILIN University, Changchun 130022, China \\ Correspondence should be addressed to Lili Zheng; zlldtq1024@163.com
}

Received 31 January 2019; Revised 10 May 2019; Accepted 7 June 2019; Published 27 June 2019

Academic Editor: Shamsunnahar Yasmin

Copyright (C) 2019 Tongqiang Ding et al. This is an open access article distributed under the Creative Commons Attribution License, which permits unrestricted use, distribution, and reproduction in any medium, provided the original work is properly cited.

\begin{abstract}
In order to ensure safe lane change and avoid traffic accidents, an effective lane change assist system is required. In a lane change assist system, it is very important to obtain the following elements in time, such as actual distance between vehicles, minimum safety distance, and warning signal. To this end, this paper analyzed four kinds of lane change angle collision scenes. Initial position, initial velocity, acceleration, heading angle, and kinematics of vehicles were used to calculate the position of potential angle collision points between lane change vehicle and obstacle vehicles. Then, actual distance model was constructed based on potential angular collision points. The minimum safety distance model was also established under the two most unfavorable conditions. In order to achieve the lane change warning, three early warning rules were formulated. We verified the validity of models and early warning rules using vehicle driving video data of Interstate 80 in California. Models and early warning rules constructed in our research can be applied to the advanced active safety systems of vehicle, such as vehicle lane change assist system and active collision early warning system, which can improve the active safety and reduce traffic accidents.
\end{abstract}

\section{Introduction}

Lane change is a relatively complicated process for driving vehicles. Drivers' improper operation during lane change may cause traffic delays and accidents. According to incomplete statistics, lane change accidents account for $4-10 \%$ of all traffic accidents [1]. Safety is affected by the type, position, speed, acceleration, and deceleration of obstacle vehicles in the present lane and the target lane. In order to ensure safe lane change and avoid angle accidents, the vehicle lane change assist system needs to calculate actual distance between lane change vehicle and obstacle vehicles and make decisions and early warning by comparing the minimum safety distance [27].

In terms of actual distance estimation between lane change vehicle and obstacle vehicles, C. L. Wang [8] processed data using statistical mean model and then applied RSSI (Received Signal Strength Indication) to estimate the distance between the two vehicles. C. Wang [9] determined actual distance between these two vehicles by calculating the distance between the microwave radar in the rear bumper of preceding vehicle and that in the preceding bumper of rear vehicle, as well as the included angle of axle wire. J. Feng et al. [10] assumed that the lateral acceleration of lane change vehicle satisfied the positive and negative trapezoidal constraints and determined the position of lane change vehicle by predicting its lane change path. Meanwhile, the distance between those vehicles is estimated by considering their length. G. Xu et al. [11] predicted the lane change trajectory to determine positions of lane change vehicles by trapezoidal lateral acceleration, spline curve, commutation trajectory of convoluted curve, polynomial function, and artificial potential field. Parameters such as the length of vehicles were considered in estimating the distance between vehicles. Y. G. Luo et al. [12] achieved actual distance measurement with other vehicles by establishing a five-time polynomial lane change trajectory tracking model for time. H. Z. Zhu et al. [13] proposed that vehicles equipped with RUPS (relative urban positioning system) can perceive their trajectory information and then perceive the relative distance between vehicles by exchanging, comparing, and identifying overlapping positions between their own and adjacent vehicle 


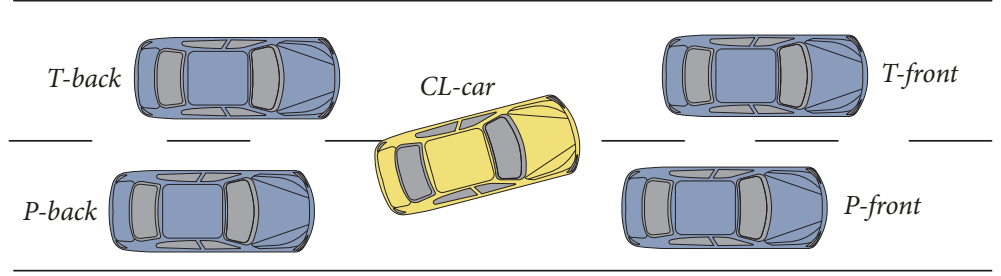

FIgURE 1: Vehicle definition.

trajectories. Y. Zhu et al. [14] detected the position of the corresponding vehicle using fish swarm algorithm. After simplifying vehicle coordinates, the corresponding actual distance was obtained by calculating their Euclidean distance. C. Olaverri-Monreal et al. [15] stereoscopically captured and processed images through the rear camera to obtain positional relationships between vehicles, with the distance between the preceding and rear vehicles calculated.

In the past studies, actual distance between lane change vehicle and obstacle vehicles was calculated by considering the coordinates of the centre points and the length of the vehicle bodies as well as the angle coordinate of lane change vehicle. In actual lane change, the collision of two vehicles depends on the space between potential angle collision points. Based on this, our research analyzed coordinates of potential angle collision points in lane change and established actual distance model for lane change vehicle and the adjacent obstacle vehicle by considering initial position, initial speed, acceleration, and heading angle of vehicle.

In terms of minimum safety distance, Q. Luo et al. [16] considered the gradual process of state and speed of preceding vehicle and established the minimum safety distance model in the motionlessness, uniform speed, and uniform deceleration of the preceding vehicle. S. M. Wang et al. [17] regarded the lateral acceleration as a sinusoidal characteristic function of time and derived the critical position of vehicle collision by derivation. Then the minimum safe distance condition was obtained to avoid vehicle collision. R. S. Tomar et al. [18] extracted the relative velocity, acceleration, and other parameters between lane change vehicle and the surrounding vehicle through support vector machine, which was used to calculate the minimum safety distance. In actual lane change, the minimum safety distance changes with the running state of vehicle, and most of the above studies cannot reflect real-time running state of vehicle [19]. A. Kanaris et al. [20] proposed that the preceding vehicle braking should be considered to calculated the minimum safety distance, so the remaining vehicles have enough time and space to adjust and avoid collision. Based on it, this paper analyzed the most unfavorable situations in lane change and combined the realtime running state of vehicle to establish the minimum safety distance model between the preceding and rear vehicles in the emergency braking of the preceding vehicle as well as the minimum safety distance model when the speed of rear vehicle is higher than that of preceding vehicle.

Different from the existing research, this paper studied functions of potential angle collision point positions under four lane change scenes to determine the actual distance model of lane change vehicles and surrounding obstacle vehicles. And then, according to the minimum safety distance, lane change risks were classified and corresponding warning rules were formulated, which assisted driver to adjust vehicle's state in time and improve vehicle's active safety.

\section{Methods}

\subsection{Relevant Vehicle Definitions and Scenes of Angle Collision during Lane Change}

2.1.1. Relevant Vehicle Definitions Involved in the Lane Change. In Figure 1, it is defined as follows: $C L$-car is the lane change vehicle; $P$-front the preceding obstacle vehicle before lane change; $P$-back the rear obstacle vehicle before lane change; T-front the preceding obstacle vehicle after lane change; $T$ back the rear obstacle vehicle after lane change.

2.1.2. Scenes of Angle Collision during Lane Change. Figure 2 shows the scenes of angle collision during lane change, that is, the angle collision of CL-car and P-front, that of CL-car and P-back, that of CL-car and T-front, and that of CL-car and T-back. Our research analyzed positions of potential angle collisions of lane change vehicles in the four scenes and established relevant models.

\subsection{Actual Distance Model between Two Vehicles}

2.2.1. Assumptions. This paper has made the following assumptions:

(1) T-back, P-back, T-front, and P-front drive straight on the respective roads without lane change, and the speed and acceleration are in a horizontal state.

(2) When the position of angle collision point is determined, lane change vehicles are regarded as a rectangle, and its coordinates are simplified to the geometric centre of the rectangle.

2.2.2. Lane Change Parameters and Coordinate Definition. Without loss of generality, modeling and analysis were carried out by taking the left lane change as an example. The lane change process was positioned by the absolute Cartesian coordinate system. The direction of the vehicle along the lane was defined as the positive direction of $\mathrm{X}$-axis; vertical to the lane and upward were defined as the positive direction of Yaxis (see Figure 3). The right edge line of the current lane is 


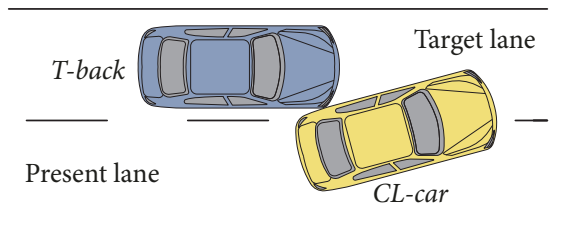

(a) The angle collision between T-back and CL-car

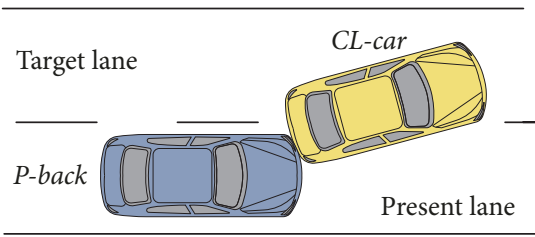

(c) The angle collision between $P$-back and $C L$ car

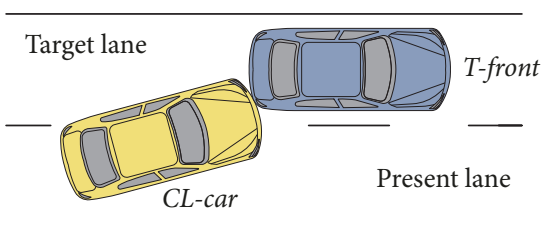

(b) The angle collision between $T$-front and $C L$ car

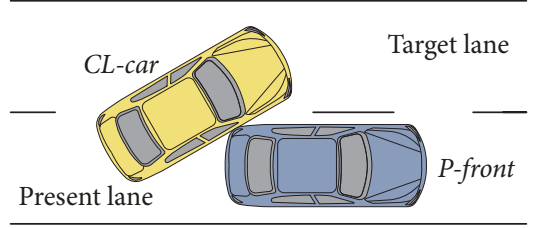

(d) The angle collision between $P$-front and $C L$ -

car

Figure 2: Scenes of angle collisions during lane change.

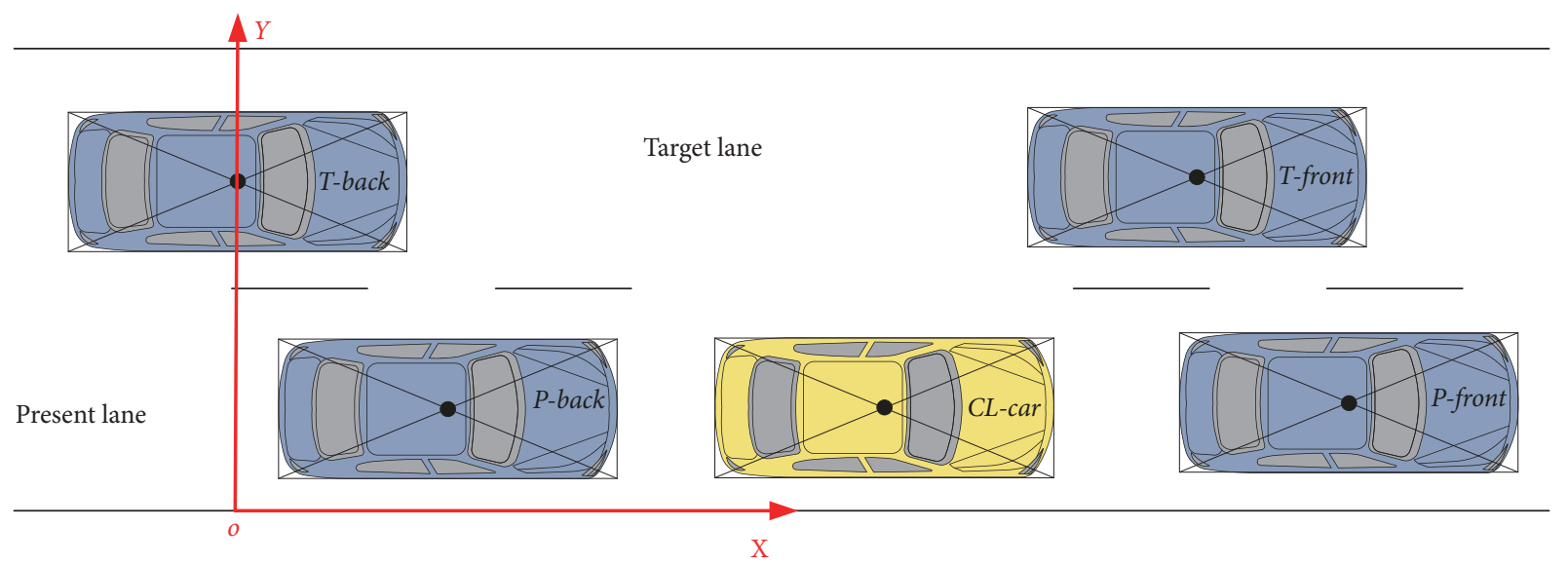

FIGURE 3: Definition of coordinate axes and coordinate origins.

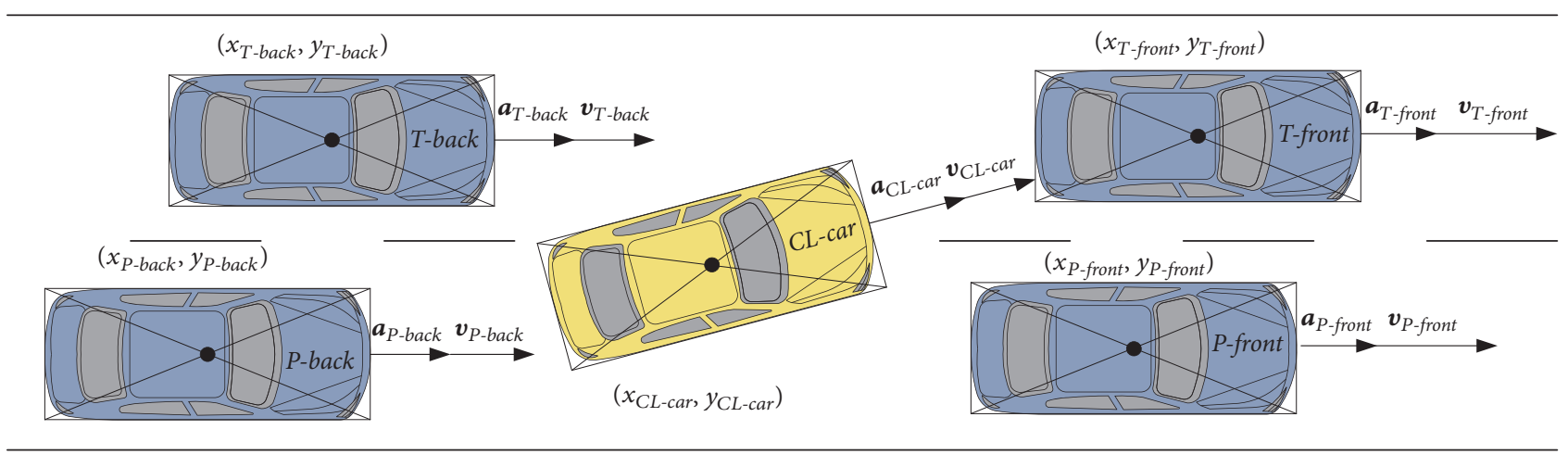

FIGURE 4: Lane change parameters.

defined as $\mathrm{X}$-axis, and Y-axis is located at the geometric centre of the end obstacle vehicle at the beginning of lane change. Then the origin of coordinates can be obtained (see Figure 3). After the definition is completed, the coordinate system does not change with the change of vehicle position during lane change.
As shown in Figure 4, the position of each vehicle is $\left(x_{j}, y_{j}\right)$, with the unit of $m ; \boldsymbol{v}_{j}$ is the speed of each vehicle, with the unit of $\mathrm{m} / \mathrm{s} ; \boldsymbol{a}_{j}$ is the acceleration of each vehicle, with the unit of $m / s^{2} ; L_{j}$ is the length of the vehicle, with the unit of $m ; W_{j}$ is the vehicle width, where $j \in[T$-back, P-back, T-front, $P$-front, $C L$-car]. 


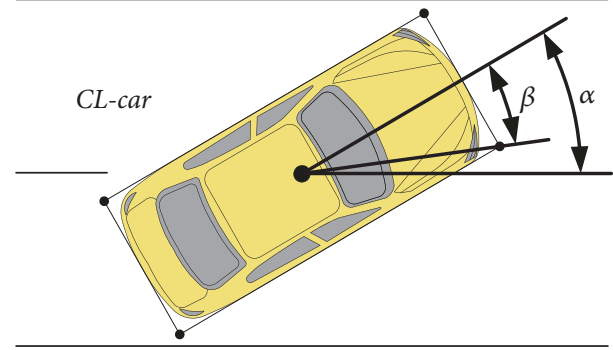

Figure 5: Angles of the lane change vehicle.

Figure 4 shows that the speed and acceleration of each vehicle are constantly changing during driving, so the start time of the lane change is defined to be zero, and the position of each vehicle can be expressed by

$$
\begin{aligned}
& x_{j}=x_{0 j}+\int\left(v_{0 j, x}+\int a_{j, x} d t\right) d t \\
& y_{j}=y_{0 j}+\int\left(v_{0 j, y}+\int a_{j, y} d t\right) d t
\end{aligned}
$$

where $x_{0 j}$ represents the initial position of the vehicle $j$ in the X direction; $v_{0 j, x}$ is the initial speed of the vehicle $j$ in the $\mathrm{X}$ direction; $a_{j, x}$ is the acceleration of the vehicle $j$ in the $\mathrm{X}$ direction. Similarly, the meanings of $y_{0 j}, v_{0 j, y}$ and $a_{j, y}$ are similar.

When the lane change vehicle CL-car is driving, the heading angle is constantly changing. In Figure $5, \alpha$ is the heading angle of vehicle; $\beta$ is the shape parameter angle of vehicle.

The angles $\alpha$ and $\beta$ can be calculated using

$$
\begin{aligned}
\alpha & =\arctan \left(\frac{v_{C L-c a r, y}}{v_{C L-c a r, x}}\right) \\
& =\arctan \left(\frac{v_{0 C L-c a r, y}+\int a_{C L-c a r, y} d t}{v_{0 C L-c a r, x}+\int a_{C L-c a r, x} d t}\right), \\
\beta & =\arctan \left(\frac{W_{C L-c a r}}{L_{C L-c a r}}\right)
\end{aligned}
$$

2.2.3. Calculation of the Preceding and Rear Angular Coordinates of Obstacle Vehicles. Figure 6 shows the preceding and rear angles of obstacle vehicles that are $B_{1, i}, B_{2, i}, B_{3, i}, B_{4, i}$. And coordinates can be calculated from the position, length, and width of obstacle vehicles (see Table 1), wherein $i \in[T$-back, P-back, T-front, P-front].

\subsubsection{Coordinate Calculation of Potential Angle Collision} Points of Lane Change Vehicles. The preceding and rear angles of lane change vehicles are defined as $A_{1}, A_{2}, A_{3}, A_{4}$, and the potential angle collision points of lane change vehicles are calculated by combining $B_{1, i}, B_{2, i}, B_{3, i}, B_{4, i}$.

(1) In the lane change scene of CL-car and P-front, the potential collision coordinates of $C L$-car are calculated as follows.
TABLE 1: Preceding and rear point coordinates of obstacle vehicles.

\begin{tabular}{lcc}
\hline $\begin{array}{l}\text { Preceding and rear angles } \\
\text { of obstacle vehicles }\end{array}$ & Abscissa & Ordinate \\
\hline$B_{1, i}$ & $x_{i}-\frac{L_{i}}{2}$ & $y_{i}-\frac{W_{i}}{2}$ \\
$B_{2, i}$ & $x_{i}-\frac{L_{i}}{2}$ & $y_{i}+\frac{W_{i}}{2}$ \\
$B_{3, i}$ & $x_{i}+\frac{L_{i}}{2}$ & $y_{i}-\frac{W_{i}}{2}$ \\
$B_{4, i}$ & $x_{i}+\frac{L_{i}}{2}$ & $y_{i}+\frac{W_{i}}{2}$ \\
\hline
\end{tabular}

Figure 7 shows that, in the lane change process 1, CL-car and $P$-front generate a potential angle collision point (1), and the potential collision position of CL-car is at point $A_{1}$. The coordinates are as follows:

$$
\begin{aligned}
& x_{A_{1}}=x_{C L-c a r}+\frac{\sqrt{L_{C L-c a r}^{2}+W_{C L-c a r}^{2}}}{2} \cos (\alpha-\beta) \\
& y_{A_{1}}=y_{C L-c a r}+\frac{\sqrt{L_{C L-c a r}^{2}+W_{C L-c a r}^{2}}}{2} \sin (\alpha-\beta)
\end{aligned}
$$

In the lane change process 2, CL-car and P-front generate a potential angle collision point (2), and the potential collision position of $C L$-car is located within $A_{1} \longrightarrow A_{2}$. The coordinates are

$$
\begin{aligned}
& x_{A_{1} \longrightarrow A_{2}}=x_{A_{2}}+\Delta x_{C L \text {-car }, P \text {-front }} \\
& y_{A_{1} \longrightarrow A_{2}}=y_{B_{2, P-\text { front }}}
\end{aligned}
$$

where

$$
\begin{aligned}
x_{A_{2}}= & x_{C L \text {-car }} \\
& -\frac{\sqrt{L_{C L-c a r}^{2}+W_{C L-c a r}^{2}}}{2} \cos (\alpha+\beta) \\
\Delta x_{C L \text {-car }, \text { P-front }}= & \Delta y_{C L \text {-car }, P-\text { front }} \cot (\alpha) \\
\Delta y_{C L \text {-car }, \text { P-front }}= & y_{B_{2, P-\text { front }}-y_{A_{2}}} \\
y_{A_{2}}= & y_{C L-\text { car }} \\
& -\frac{\sqrt{L_{C L-c a r}^{2}+W_{C L-c a r}^{2}}}{2} \sin (\alpha+\beta)
\end{aligned}
$$

(2) In the lane change scene of CL-car and P-back, the potential collision position coordinates of $C L$-car are calculated as follows.

Figure 8 shows that in the lane change process $1, C L$ car and $P$-back generate a potential angle collision point (1), and the potential collision position of $C L$-car is at $A_{3}$. The coordinates are

$$
x_{A_{3}}=x_{C L-c a r}-\frac{\sqrt{L_{C L-c a r}^{2}+W_{C L-c a r}^{2}}}{2} \cos (\alpha-\beta)
$$




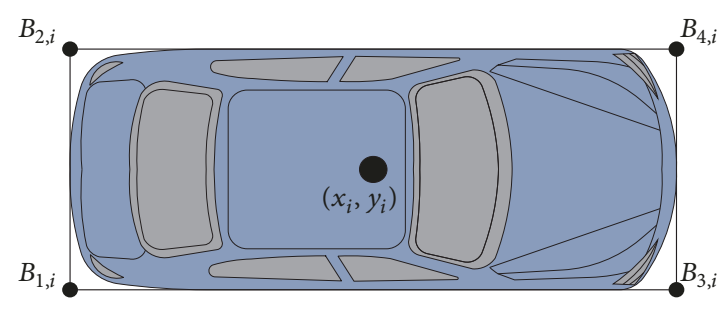

Figure 6: Preceding and rear angles of obstacle vehicles.

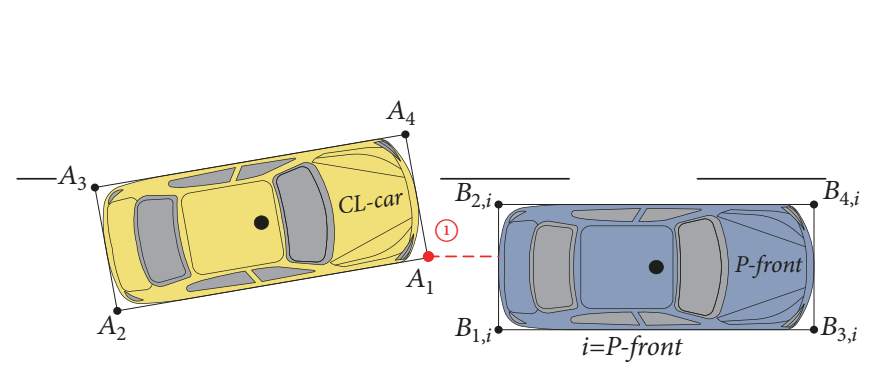

Lane change process 1

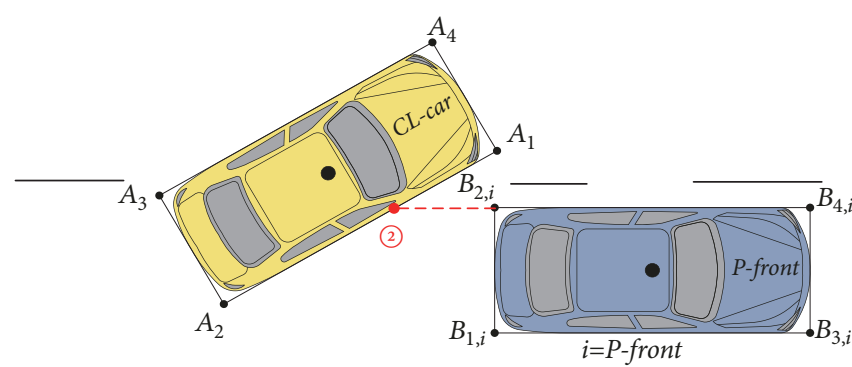

Lane change process 2

Figure 7: Potential angle collision point positions in different lane change processes between CL-car and P-front.

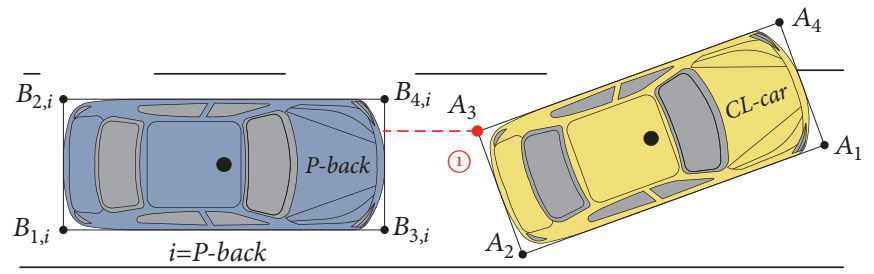

Lane change process 1

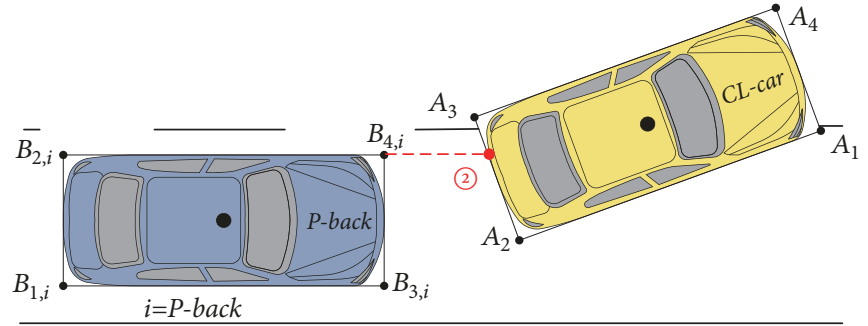

Lane change process 2

FIGURE 8: Potential angle collision point positions in different lane change processes between CL-car and P-back.

$$
y_{A_{3}}=y_{C L-c a r}-\frac{\sqrt{L_{C L-c a r}^{2}+W_{C L-c a r}^{2}}}{2} \sin (\alpha-\beta)
$$

As shown in Figure 8, in the lane change process 2, CL-car and $P$-back generate a potential angle collision point (2), and the potential collision position of $C L$-car is located within $A_{2} \longrightarrow$ $A_{3}$. The coordinates are

$$
\begin{aligned}
& x_{A_{2} \rightarrow A_{3}}=x_{A_{2}}-\Delta x_{P \text {-back,CL-car }} \\
& y_{A_{2} \rightarrow A_{3}}=y_{B_{4, P} \text {-back }}
\end{aligned}
$$

where

$$
\begin{aligned}
& \Delta x_{P-\text { back }, C L-c a r}=\Delta y_{P-\text { back }, C L-c a r} \tan (\alpha) \\
& \Delta y_{P-\text { back }, C L-c a r}=y_{B_{4, \text { P-back }}}-y_{A_{2}}
\end{aligned}
$$

(3) In the lane change scene of CL-car and T-front, the potential collision position coordinates of $C L$-car are calculated as follows.

As shown in Figure 9, in the lane change process 1, CLcar and $T$-front generate a potential angle collision point (1), and the potential collision position of CL-car is located within $A_{4} \longrightarrow A_{1}$. The coordinates are

$$
\begin{aligned}
& x_{A_{4} \longrightarrow A_{1}}=x_{A_{1}}-\Delta x_{C L \text {-car }, T \text {-front }} \\
& y_{A_{4} \longrightarrow A_{1}}=y_{B_{1, T-\text { front }}}
\end{aligned}
$$

where

$$
\begin{aligned}
& \Delta x_{C L \text {-car }, T \text {-front }}=\Delta y_{C L \text {-car }, T \text {-front }} \tan (\alpha) \\
& \Delta y_{C L \text {-car }, T \text {-front }}=y_{B_{1, T-\text { front }}}-y_{A_{1}}
\end{aligned}
$$

In the lane change process $2, C L$-car and T-front generate a potential angle collision point (2); the potential collision position of $C L$-car is at $A_{1}$; its coordinate is $\left(x_{A_{1}}, y_{A_{1}}\right)$.

(4) In the lane change scene of CL-car and T-back, the potential collision position coordinates of CL-car are calculated as follows.

Figure 10 shows that, in the lane change process $1, C L$ car and T-back generate a potential angle collision point (1), 
Lane change process 1

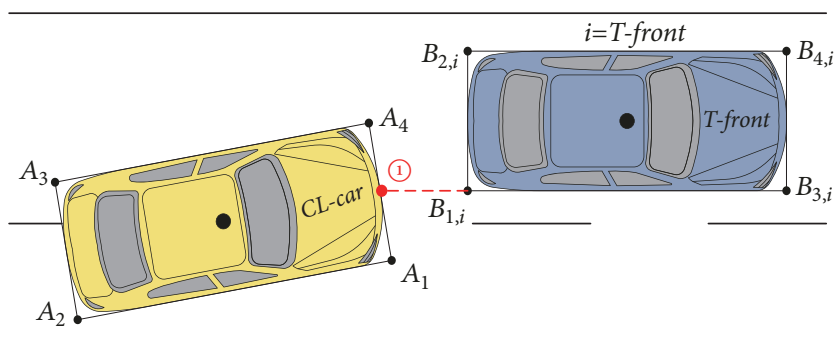

Lane change process 2

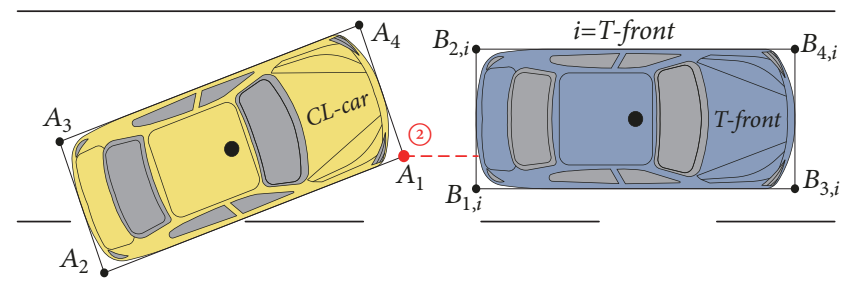

FIgURE 9: Potential angle collision point positions in different lane change processes between CL-car and T-front.

Lane change process 1

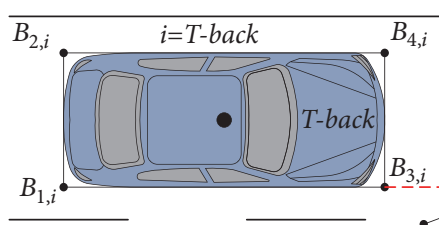

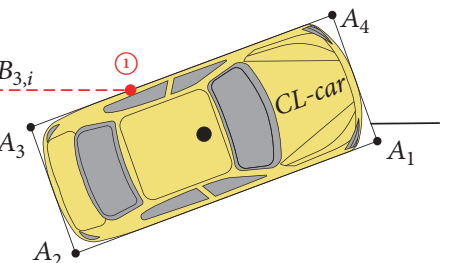

Lane change process 2

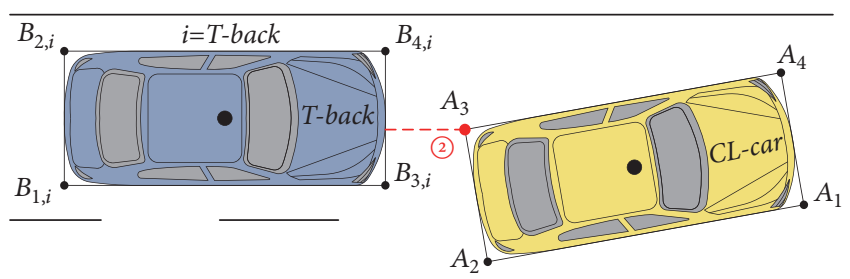

FIGURE 10: Potential angle collision point positions in different lane change processes between CL-car and T-back.

and the potential collision position of $C L$-car is located within $A_{3} \longrightarrow A_{4}$. The coordinates are

$$
\begin{aligned}
& x_{A_{3} \rightarrow A_{4}}=x_{A_{4}}-\Delta x_{T-\text { back,CL-car }} \\
& y_{A_{3} \longrightarrow A_{4}}=y_{B_{3, T-\text { back }}}
\end{aligned}
$$

where

$$
\begin{aligned}
x_{A_{4}}= & x_{C L-c a r} \\
& +\frac{\sqrt{L_{C L-c a r}^{2}+W_{C L-c a r}^{2}}}{2} \cos (\alpha+\beta) \\
\Delta x_{T-\text { back }, C L-c a r}= & y_{T-\text { back,CL-car }} \cot (\alpha) \\
\Delta y_{T-\text { back,CL-car }}= & y_{A_{4}}-y_{B_{3, T-\text { back }}} \\
y_{A_{4}}= & y_{T-\text { back }} \\
& +\frac{\sqrt{L_{C L-c a r}^{2}+W_{C L-c a r}^{2}}}{2} \sin (\alpha+\beta)
\end{aligned}
$$

Figure 10 shows, in the lane change process 2, CL-car and $T$-back generate a potential angle collision point 2; the potential collision position of $C L$-car is at $A_{3}$; its coordinate is $\left(x_{A_{3}}, y_{A_{3}}\right)$.

2.2.5. Determination of Actual Distance. According to the preceding and rear angular coordinates of obstacle vehicles and the coordinates of potential angle collision points of lane change vehicle, we calculate actual distance between lane change vehicle and obstacle vehicles during the twostage lane change in four scenes. Figure 11 shows that when

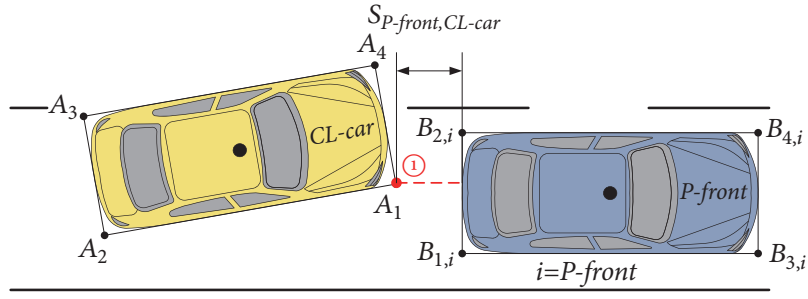

Figure 11: Actual distance diagram.

$y_{B_{1, P \text {-front }}}<y_{A_{1}}<y_{B_{2, P-\text { front }}}$, the potential angle collision position of $C L$-car is at $A_{1}$, and the actual distance $S_{P-\text { front,CL-car }}$ between the two vehicles can be calculated by

$$
S_{P-\text { front }, \text { CL-car }}=x_{B_{2, \text { P-front }}}-x_{A_{1}}
$$

Similarly, actual distance between lane change vehicle and obstacle vehicles in other scenes can be obtained (see Table 2).

2.3. Minimum Safety Distance Model. For the assisted driving system, after measuring actual distance between lane change vehicle and obstacle vehicles, it is necessary to determine the corresponding minimum safety distance for safe lane change. Decision and early warning are made by comparison [21] The minimum safe distance refers to the necessary separation distance between the rear and preceding vehicles to avoid collision.

In lane change, there are two most unfavorable scenes: one is emergency braking of the preceding vehicle and the other is the speed of rear vehicle which is greater than that of preceding vehicle. For the first type, when preceding vehicle 
TABLE 2: Actual distance during the two-stage lane change in four scenes.

\begin{tabular}{|c|c|c|c|c|}
\hline $\begin{array}{l}\text { Angle collision } \\
\text { scene }\end{array}$ & Angle collision point & Conditions for angle collision point & Actual distance & Remarks \\
\hline \multirow{2}{*}{$\begin{array}{l}C L \text {-car and } \\
P \text {-front }\end{array}$} & (1) & $y_{B_{1, P-\text { front }}}<y_{A_{1}}<y_{B_{2, P-\text { front }}}$ & $x_{B_{2, P \text {-front }}}-x_{A_{1}}$ & \multirow{2}{*}{$S_{P \text {-front }, \text { CL-car }}$} \\
\hline & (2) & $y_{A_{1}}>y_{B_{2, P-\text { front }}}, y_{A_{2}}<y_{B_{2, P-\text { front }}}$ & $x_{B_{2, P-\text { front }}}-x_{A_{1} \longrightarrow A_{2}}$ & \\
\hline \multirow{2}{*}{$\begin{array}{l}\text { CL-car and } \\
P \text {-back }\end{array}$} & (1) & $y_{B_{3, P-\text { back }}}<y_{A_{3}}<y_{B_{4, P} \text {-back }}$ & $x_{A_{3}}-x_{B_{4, P-b a c k}}$ & \multirow{2}{*}{$S_{C L-c a r, P-b a c k}$} \\
\hline & (2) & $y_{A_{3}}>y_{B_{4, P \text {-back }}}, y_{A_{2}}<y_{B_{4, \text { P-back }}}$ & $x_{A_{2} \rightarrow A_{3}}-x_{B_{4, P-\text { back }}}$ & \\
\hline \multirow{2}{*}{$\begin{array}{l}C L \text {-car and } \\
T \text {-front }\end{array}$} & (1) & $y_{A_{1}}<y_{B_{1, T-\text { front }}}, y_{A_{4}}>y_{B_{1, T-\text { front }}}$ & $x_{B_{1, T-\text { front }}}-x_{A_{4} \longrightarrow A_{1}}$ & \multirow{2}{*}{$S_{T-\text { front }, C L-c a r}}$, \\
\hline & (2) & $y_{B_{2, T-\text { front }}}>y_{A_{1}}>y_{B_{1, T-\text { front }}}$ & $x_{B_{1, T-\text { front }}}-x_{A_{1}}$ & \\
\hline \multirow{2}{*}{$\begin{array}{l}\text { CL-car and } \\
\text { T-back }\end{array}$} & (1) & $y_{A_{3}}<y_{B_{3, T-b a c k}}, y_{A_{4}}>y_{B_{3, T-b a c k}}$ & $x_{A_{3} \rightarrow A_{4}}-x_{B_{3, T-b a c k}}$ & \multirow{2}{*}{$S_{C L-c a r, T-b a c k}$} \\
\hline & (2) & $y_{B_{4, T-b a c k}}>y_{A_{3}}>y_{B_{3, T-b a c k}}$ & $x_{A_{3}}-x_{B_{3, T} \text {-back }}$ & \\
\hline
\end{tabular}

is parked under emergency braking, the rear vehicle should adjust its deceleration and speed to make the vehicle stop within the shortest distance for safety [22]. The second type is that, during lane change of continuous driving, the rear vehicle needs to decelerate to the same speed as preceding vehicle for safety.

In view of the above-mentioned most unfavorable scenes, based on kinematics principle of vehicle, this paper established the minimum safety distance model between preceding and rear vehicles when the preceding vehicle is in emergency braking and the minimum safety distance model between preceding and rear vehicles when the rear vehicle is adjusted to the same speed as the preceding vehicle.

\subsubsection{Minimum Safety Distance Model between the Preceding} and Rear Vehicles during Emergency Braking of the Preceding Vehicle. The vehicle braking time consists of driver response time, brake coordination time, brake acceleration growth time, and continuous braking time [23], wherein vehicle tires, suspension, and ground have the impact on brake coordination time $[24,25]$. The sum of driver response time and brake coordination time is generally $0.8-1.0 \mathrm{~s}[26,27]$. The deceleration build-up time of the preceding vehicle is generally 0.1 to $0.2 \mathrm{~s}$, and $t_{\text {front,c }}$ is the braking time of preceding vehicle, with the unit of $s . t_{\text {back }}$ is the deceleration build-up time of the rear car, generally 0.1-0.2 s, and $t_{b a c k, c}$ is the rear brake time, with the unit of $s$ [28]. The braking principles of different vehicles are different [29, 30], and the maximum braking force is not exactly the same. The maximum average braking acceleration in the horizontal direction of the vehicle during emergency braking is determined as $7 \mathrm{~m} / \mathrm{s}^{2}[27,31]$. Since the driver of the rear vehicle takes action after the preceding vehicle brakes, the change of the braking acceleration of rear vehicle will be delayed relative to the preceding vehicle. When the minimum safety distance $L B_{b a c k \text {, front }}$ is calculated, the acceleration changes of preceding and rear vehicles are shown in Figures 12 and 13.

Then the braking distance $L_{\text {back }}$ of the rear vehicle is

$$
\begin{aligned}
L_{\text {back }}= & v_{\text {back,x }}\left(t_{r 1}+t_{r 2}+\frac{t_{\text {back }}}{2}\right)-\frac{1}{24} a_{\text {maxback }, x} t_{b a c k}^{2} \\
& +\frac{v_{\text {back,x }}^{2}}{2 a_{\text {maxback }, x}}
\end{aligned}
$$

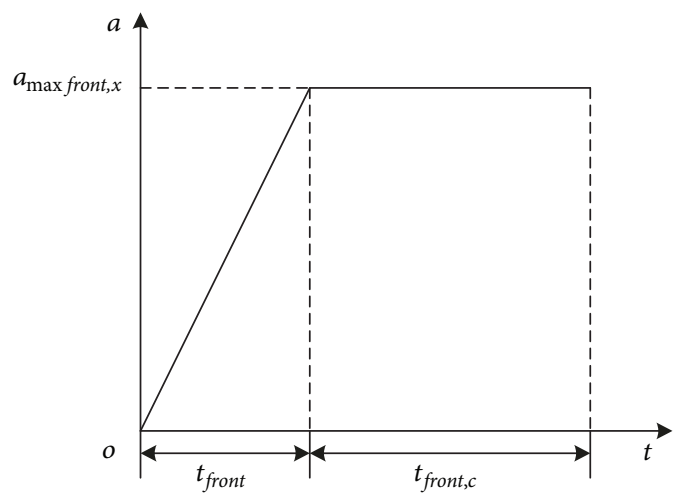

FIGURE 12: Braking deceleration changes of preceding vehicle.

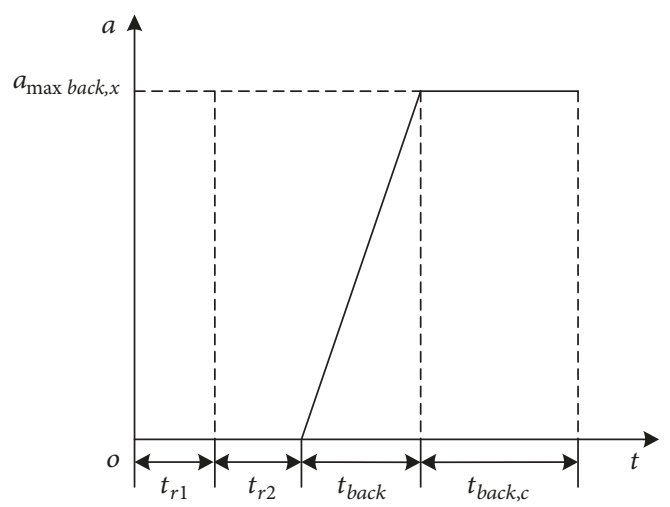

FIGURE 13: Braking deceleration changes of rear vehicle.

where $v_{b a c k, x}$ represents the speed of the rear vehicle in $\mathrm{X}$ direction; $a_{\max b a c k, x}$ is the maximum braking acceleration of the rear vehicle in $\mathrm{X}$ direction.

Similarly,

$$
\begin{aligned}
L_{C L-c a r, b a c k}= & v_{C L-c a r, x}\left(t_{r 1}+t_{r 2}+\frac{t_{\text {back }}}{2}\right) \\
& -\frac{1}{24} a_{\max C L-c a r, x} t_{\text {back }}^{2}+\frac{v_{C L-c a r, x}^{2}}{2 a_{\max C L-c a r, x}}
\end{aligned}
$$




$$
\begin{aligned}
L_{P \text {-back }}= & v_{P \text {-back }, x}\left(t_{r 1}+t_{r 2}+\frac{t_{\text {back }}}{2}\right) \\
& -\frac{1}{24} a_{\max P \text {-back, } x} t_{\text {back }}^{2}+\frac{v_{P \text {-back,x }}^{2}}{2 a_{\max P \text {-back }, x}} \\
L_{T-b a c k}= & v_{T-b a c k, x}\left(t_{r 1}+t_{r 2}+\frac{t_{\text {back }}}{2}\right) \\
& -\frac{1}{24} a_{\text {maxT-back, } x} t_{\text {back }}^{2}+\frac{v_{T-b a c k, x}^{2}}{2 a_{\text {maxT-back, } x}}
\end{aligned}
$$

The braking distance of the preceding vehicle is

$$
\begin{aligned}
L_{\text {front }}= & \frac{1}{2} v_{\text {front }, x} t_{\text {front }}-\frac{1}{24} a_{\max \text { front }, x} t_{\text {front }}^{2} \\
& +\frac{v_{\text {front }, x}^{2}}{2 a_{\max \text { front }, x}}
\end{aligned}
$$

where $v_{\text {front }, x}$ represents the speed of the preceding vehicle in $\mathrm{X}$ direction; $a_{\max \text { front }, x}$ is the maximum braking acceleration of the preceding vehicle in $\mathrm{X}$ direction.

Similarly,

$$
\begin{aligned}
& L_{P-\text { front }}=\frac{1}{2} v_{P-\text { front }, x} t_{\text {front }}-\frac{1}{24} a_{\max P-\text { front }, x} t_{\text {front }}^{2} \\
& +\frac{v_{P-\text { front }, x}^{2}}{2 a_{\max P-\text { front }, x}} \\
& L_{\text {T-front }}=\frac{1}{2} v_{T \text {-front }, x} t_{\text {front }}-\frac{1}{24} a_{\max T \text {-front }, x} t_{\text {front }}^{2} \\
& +\frac{v_{T-\text { front }, x}^{2}}{2 a_{\max T-\text { front }, x}} \\
& L_{C L-c a r, \text { front }}=\frac{1}{2} v_{C L-c a r, x} t_{\text {front }}-\frac{1}{24} a_{\text {maxCL-car }, x} t_{\text {front }}^{2} \\
& +\frac{v_{C L-c a r, x}^{2}}{2 a_{\max C L-c a r, x}}
\end{aligned}
$$

The minimum safety distance $L B_{\text {back, front }}$ under emergency braking of the preceding vehicle is

$$
L B_{\text {back, front }}=L_{\text {back }}-L_{\text {front }}
$$

Similarly,

$$
\begin{aligned}
& L B_{C L \text {-car }, P \text {-front }}=L_{C L \text {-car,back }}-L_{P \text {-front }} \\
& L B_{C L \text {-car,T-front }}=L_{C L \text {-car,back }}-L_{T \text {-front }} \\
& L B_{P \text {-back,CL-car }}=L_{P \text {-back }}-L_{C L \text {-car, front }} \\
& L B_{T \text {-back,CL-car }}=L_{T \text {-back }}-L_{C L \text {-car, front }}
\end{aligned}
$$

2.3.2. Minimum Safety Distance Model between Preceding and Rear Vehicles When the Rear Vehicle Is Adjusted to the Same Speed as the Preceding Vehicle. If the speed $v_{\text {front }, x}$ of the preceding vehicle is greater than the speed $v_{b a c k, x}$ of the rear vehicle, the actual distance between the two vehicles increases with time, and no angle collision and rear-end collision occur. Therefore, the minimum safety distance $L S_{\text {back, front }}$ is zero [32]. If the speed $v_{\text {front,x }}$ of the preceding vehicle is less than the speed $v_{b a c k, x}$ of the rear vehicle, the minimum safe distance is

$$
L S_{\text {back,front }}=\frac{v_{\text {back,x }}^{2}-v_{\text {front }, x}^{2}}{2 a_{\max b a c k, x}}
$$

Similarly,

$$
\begin{aligned}
& L S_{C L \text {-car }, P \text {-front }} \\
& \quad= \begin{cases}0 & v_{P-\text { front }, x} \geq v_{C L \text {-car }, x} \\
\frac{v_{C L-c a r, x}^{2}-v_{P-\text { front }, x}^{2}}{2 a_{\max C L-c a r, x}} & v_{P-\text { front }, x}<v_{C L \text {-car }, x}\end{cases}
\end{aligned}
$$

$$
\begin{aligned}
& L S_{C L-c a r, T-f r o n t} \\
& = \begin{cases}0 & v_{T-\text { front }, x} \geq v_{C L \text {-car }, x} \\
\frac{v_{C L \text {-car }, x}^{2}-v_{T-\text { front }, x}^{2}}{2 a_{\max C L-c a r, x}} & v_{T-\text { front }, x}<v_{C L \text {-car }, x}\end{cases} \\
& L S_{P-b a c k, C L-c a r} \\
& = \begin{cases}0 & v_{C L-c a r, x} \geq v_{P-b a c k, x} \\
\frac{v_{P-b a c k, x}^{2}-v_{C L-c a r, x}^{2}}{2 a_{\max P-b a c k, x}} & v_{C L-c a r, x}<v_{P-b a c k, x}\end{cases} \\
& L S_{\text {T-back,CL-car }} \\
& = \begin{cases}0 & v_{C L-c a r, x} \geq v_{T-b a c k, x} \\
\frac{v_{T-b a c k, x}^{2}-v_{C L-c a r, x}^{2}}{2 a_{\max T-b a c k, x}} & v_{C L \text {-car }, x}<v_{T \text {-back,x }}\end{cases}
\end{aligned}
$$

2.4. Definition of Early Warning Rules. According to the position of the potential angle collision point, we can obtain the actual distance $S_{\text {back, front }}$ between the rear vehicle and preceding vehicle, the minimum safety distance $L B_{b a c k \text {, front }}$ under the emergency braking of the preceding vehicle, and the minimum safety distance $L S_{\text {back, front }}$ between preceding and rear vehicles when the rear vehicle is adjusted to the same speed as the preceding vehicle. The comparison of $S_{\text {back, front }}$, $L B_{\text {back, front }}$, and $L S_{\text {back, front }}$ divides the early warning into no early warning, mild early warning, and high early warning, and the colors of the early warning lights are green, yellow, and red, respectively. Specific definitions are shown in Table 3.

\section{Result and Discussion}

3.1. Introduction to Data Sources. The model validation data in our research was obtained from vehicle travel video data of Interstate 80 in Emeryville, California, collected by the 
TABLE 3: Introduction to early warning rules.

\begin{tabular}{|c|c|c|c|}
\hline $\begin{array}{l}\text { Early warning } \\
\text { rule }\end{array}$ & Condition & Status description & Display color \\
\hline $\begin{array}{l}\text { No early } \\
\text { warning } \\
\end{array}$ & $S_{\text {back,front }}>L B_{\text {back,front }}$ & No need to adjust the speed & \\
\hline $\begin{array}{l}\text { Mild early } \\
\text { warning }\end{array}$ & $\begin{array}{c}L S_{\text {back,front }}<S_{\text {back, front }} \leq \\
L B_{\text {back, front }}\end{array}$ & $\begin{array}{l}\text { If the preceding vehicle does not } \\
\text { take emergency braking, the } \\
\text { distance between the vehicles } \\
\text { meets the safety needs. }\end{array}$ & \\
\hline $\begin{array}{l}\text { Severe early } \\
\text { warning }\end{array}$ & $S_{\text {back,front }} \leq L S_{\text {back,front }}$ & $\begin{array}{l}\text { When the speed of the vehicle is } \\
\text { not adjusted in time, a angle } \\
\text { collision accident will occur. }\end{array}$ & \\
\hline
\end{tabular}

TABle 4: Vehicle basic data.

\begin{tabular}{lccccc}
\hline Vehicle & $L_{j}(m)$ & $W_{j}(m)$ & $x_{0 j}(m)$ & $y_{0 j}(m)$ & $v_{0 j, x}(m / s)$ \\
\hline CL-car & 4.20624 & 2.22504 & 12.8784096 & 2.2856216 & 11.3011712 \\
P-front & 18.19656 & 2.59080 & 41.1053280 & 1.3757936 & 8.9631520 \\
T-front & 4.05384 & 2.07264 & 17.5287432 & 5.8575728 & 16.6254176 \\
P-back & 5.15112 & 1.79832 & 1.67365680 & 1.8046472 & 11.0150656 \\
T-back & 4.81584 & 2.10312 & 0 & 6.0776384 & 15.6151072 \\
\hline
\end{tabular}

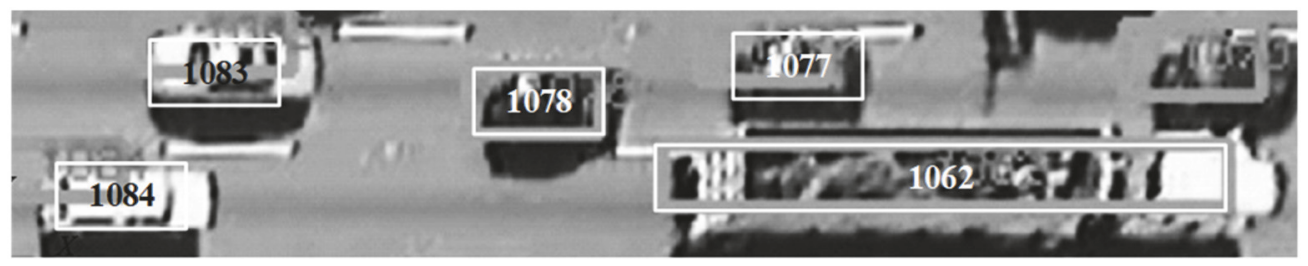

FIGURE 14: Data extraction scene.

Berkeley Highway Laboratory, from 4:00 pm to 4:15 pm on April 13, 2005. The US federal highway management software NGSIM was used to transcribe video data at 10 frames per second and then obtain data such as the length, width, coordinates, acceleration, and other data of vehicle in each frame.

3.2. Model Verification Parameters and Preprocessing. This paper analyzed the lane change video of the vehicle 1078, which considers preceding and rear obstacle vehicles before and after lane change. Thus, vehicle 1078 is defined as CL-car, vehicle 1062 as $P$-front, vehicle 1077 as T-front, vehicle 1083 as T-back, and vehicle 1084 as P-back (see Figure 14). According to the definition of coordinates origin and coordinate axis in Section 2.2.2, at the beginning of lane change, the end obstacle vehicle is vehicle 1083, so Y-axis in coordinate system is located at the geometric centre of vehicle 1083, and X-axis is on the right edge of the lane where vehicle 1084 and vehicle 1062 are located. Table 4 shows the data involved in model validation.

Since the data is extracted by frame, it is necessary to fit the acceleration of vehicle. MATLAB is used to determine the acceleration of lane change vehicle and obstacle vehicles. Figures 15-20 show the vehicle acceleration versus time

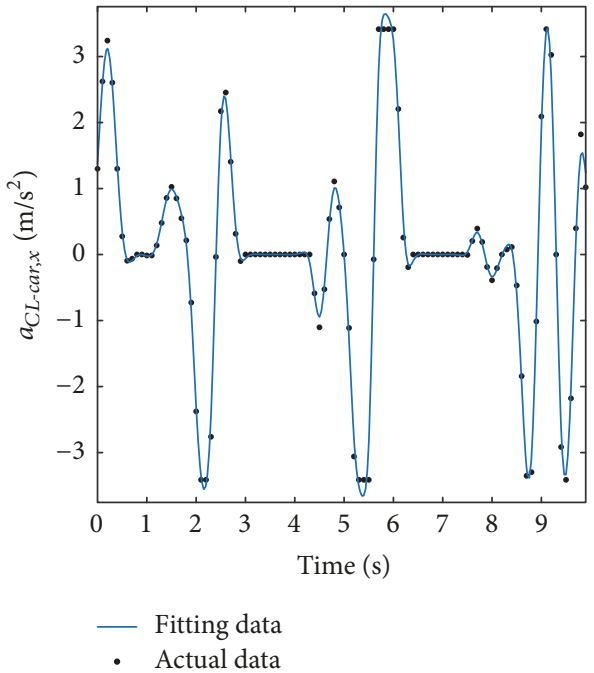

FIGURE 15: Acceleration curve of CL-car in the positive direction of $\mathrm{X}$-axis with time.

curve, and Table 5 shows the corresponding fitting effect indicators. 


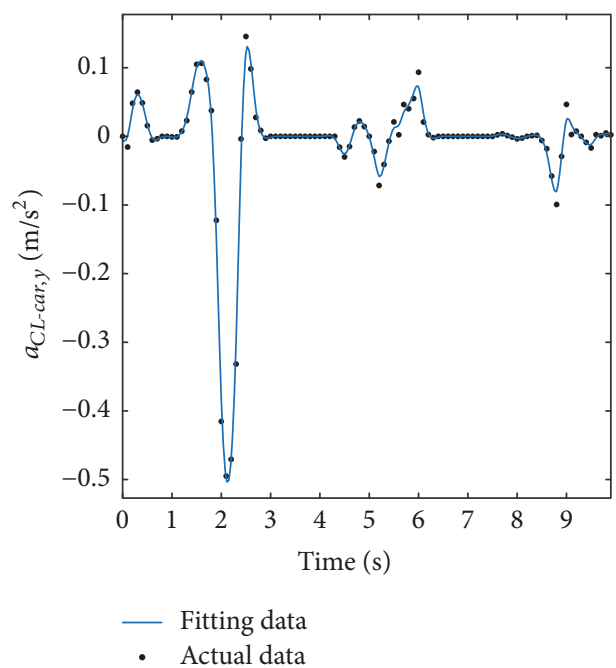

FIgURE 16: Acceleration curve of $C L$-car in the positive direction of Y-axis with time.

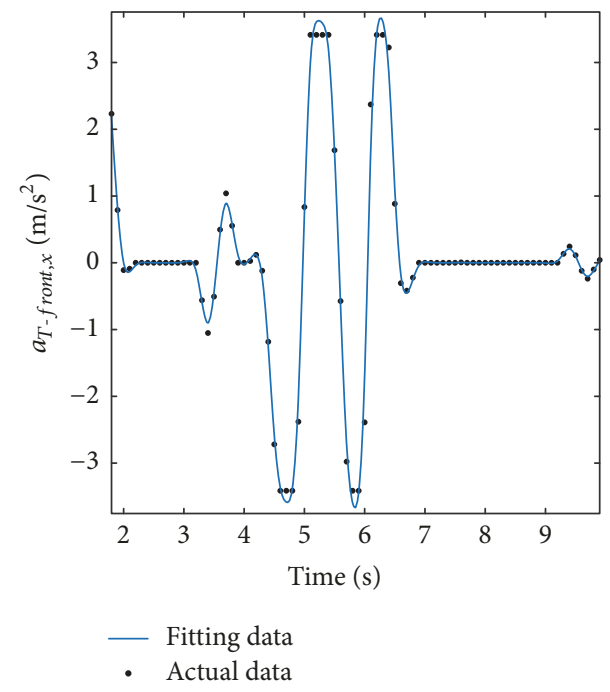

FIGURE 17: Acceleration curve of T-front in the positive direction of $\mathrm{X}$-axis with time.

TABLE 5: Fitting effect indicators.

\begin{tabular}{lccc}
\hline & R-square & Adjusted R-square & RMSE \\
\hline$a_{C L \text {-car }, x}$ & 0.993 & 0.982 & 0.221 \\
$a_{C L \text {-car }, y}$ & 0.991 & 0.978 & 0.014 \\
$a_{P-\text { front }, x}$ & 0.993 & 0.981 & 0.184 \\
$a_{T-\text { front }, x}$ & 0.990 & 0.974 & 0.245 \\
$a_{P-\text { back }, x}$ & 0.994 & 0.985 & 0.156 \\
$a_{T-\text { back }, x}$ & 0.994 & 0.983 & 0.162 \\
\hline
\end{tabular}

3.3. Early Warning Analysis. According to the established actual distance model, minimum safety distance model, and early warning rule definition, this paper drew the time variation curves of actual distance and the minimum safety distance between the lane change vehicle 1078 and its adjacent obstacle vehicles, and the curve of vehicles speed changes

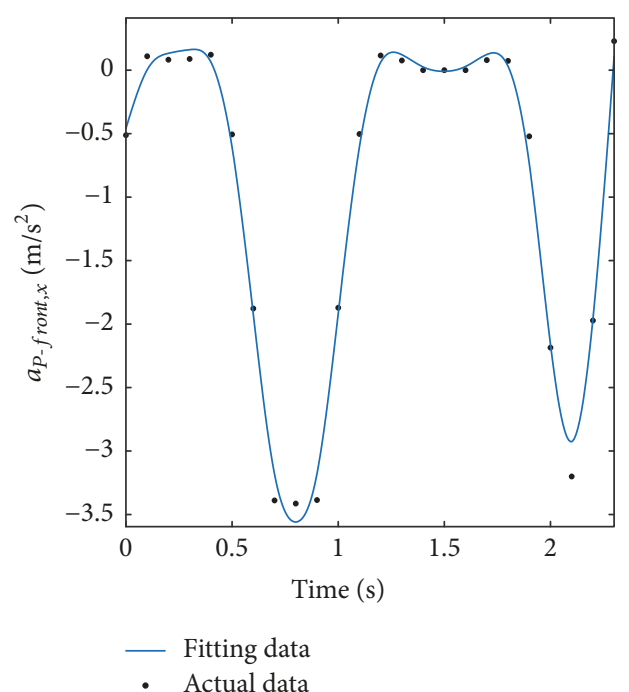

FIgURE 18: Acceleration curve of $P$-front in the positive direction of $\mathrm{X}$-axis with time.

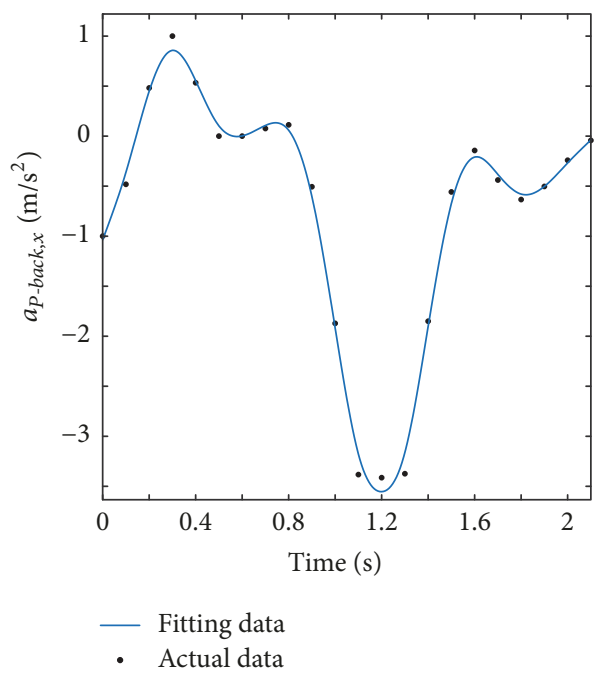

FIgURE 19: Acceleration curve of P-back in the positive direction of $\mathrm{X}$-axis with time.

with time (see Figures 21-24). Through comparing with video recording, the actual distance variation curve between vehicles is consistent with the change of the distance between vehicles in the video, and the validity of the model constructed in the work is verified. At the same time, according to early warning rules, warnings and prompts can be given to potential conflict vehicles. The early warning analysis is as follows.

3.3.1. Early Warning Analysis of CL-car and P-front. Figure 21 shows in the early stage of the potential angle collision point (1) that $v_{C L \text {-car }, x}(t)$ is always greater than $v_{P \text {-front }, x}(t)$, resulting in the decrease of $S_{C L \text {-car }, P \text {-front }}(t)$ and the increase of $L S_{C L \text {-car,P-front }}(t)$ and $L B_{C L \text {-car, } P \text {-front }}(t)$. Since $S_{C L \text {-car,P-front }}(t)$ is always greater than $L B_{C L \text {-car,P-front }}(t)$ and $L S_{C L \text {-car,P-front }}(t)$ 


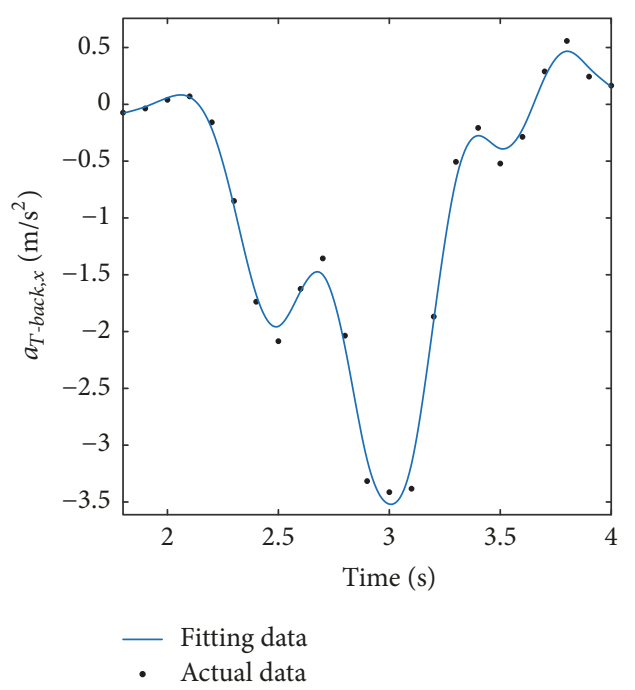

Figure 20: Acceleration curve of T-back in the positive direction of $\mathrm{X}$-axis with time.

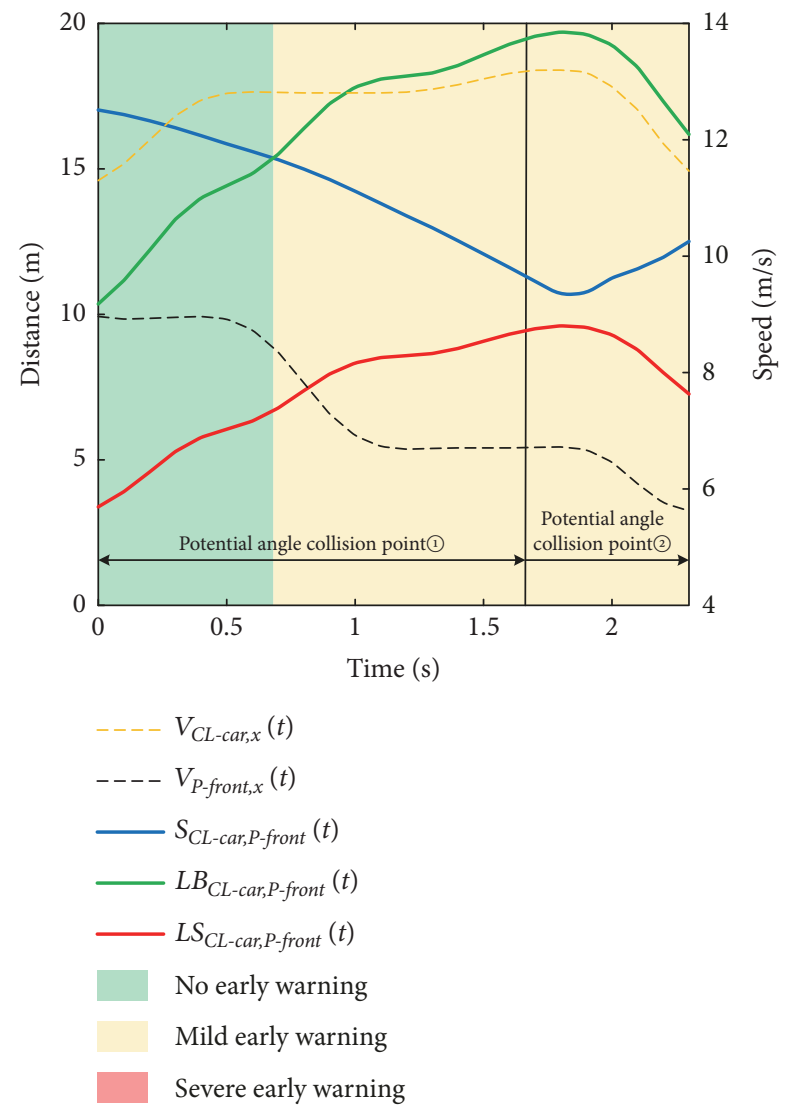

FIgURE 21: Distance and speed change between $C L$-car and $P$-front.

at this stage, which satisfies the safety conditions, CL-car needs no early warning.

In the middle and late stages of the potential angle collision point (1), $v_{C L-c a r, x}(t)$ is always greater than $v_{P-\text { front }, x}(t)$, resulting in the decrease of $S_{C L \text {-car,P-front }}(t)$ and the increase of $L S_{C L-c a r, P-\text { front }}(t)$ and $L B_{C L \text {-car,P-front }}(t)$. $S_{C L \text {-car,P-front }}(t)$

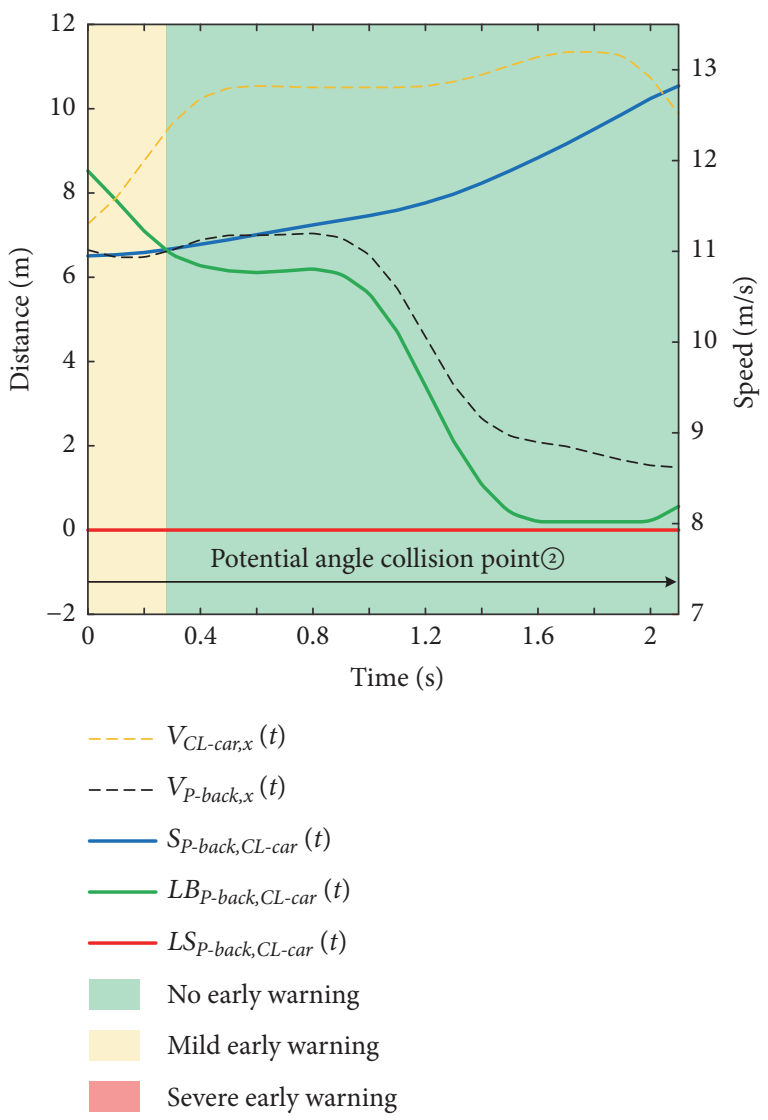

Figure 22: Distance and speed change between P-back and CL-car.

is always greater than $L S_{C L \text {-car,P-front }}(t)$ and less than $L B_{C L-c a r, P-f r o n t}(t)$. Then, CL-car should be given the mild early warning and pay attention to the emergency brake of preceding vehicle with appropriate deceleration.

During the potential angle collision point (2), $v_{C L-c a r, x}(t)$ is always greater than $v_{P-\text { front }, x}(t)$, but the change of potential angle collision point increases $S_{C L-c a r, P-f r o n t}(t)$ and decreases $L S_{C L-c a r, P-f r o n t}(t)$ and $L B_{C L-c a r, P-f r o n t}(t)$. Since $S_{C L-c a r, P-\text { front }}(t)$ is always greater than $L S_{C L \text {-car,P-front }}(t)$ and less than $L B_{C L \text {-car, } P \text {-front }}(t), C L$-car should be given mild early warning and pay attention to the emergency braking of preceding vehicle with appropriate deceleration. When there is no potential angle collision point between two vehicles, the early warning is terminated.

3.3.2. Early Warning Analysis of CL-car and P-back. Figure 22 shows that since $C L$-car travels to the left road edge line in the lane, there is potential angle collision point (2) between vehicles instead of potential angle collision point (1). In the early stage of potential angle collision point (2), $S_{P \text {-back,CL-car }}(t)$ is less than $L B_{P \text {-back,CL-car }}(t)$ and greater than $L S_{P \text {-back,CL-car }}(t)$. $P$-back can be given mild early warning and pay attention to the emergency braking of preceding vehicle with appropriate deceleration. The speed $v_{C L-c a r, x}(t)$ of the lane change vehicle $C L$-car is basically in increase trend; there is a slight increase for $v_{P-b a c k, x}(t)$ in the early period, but 


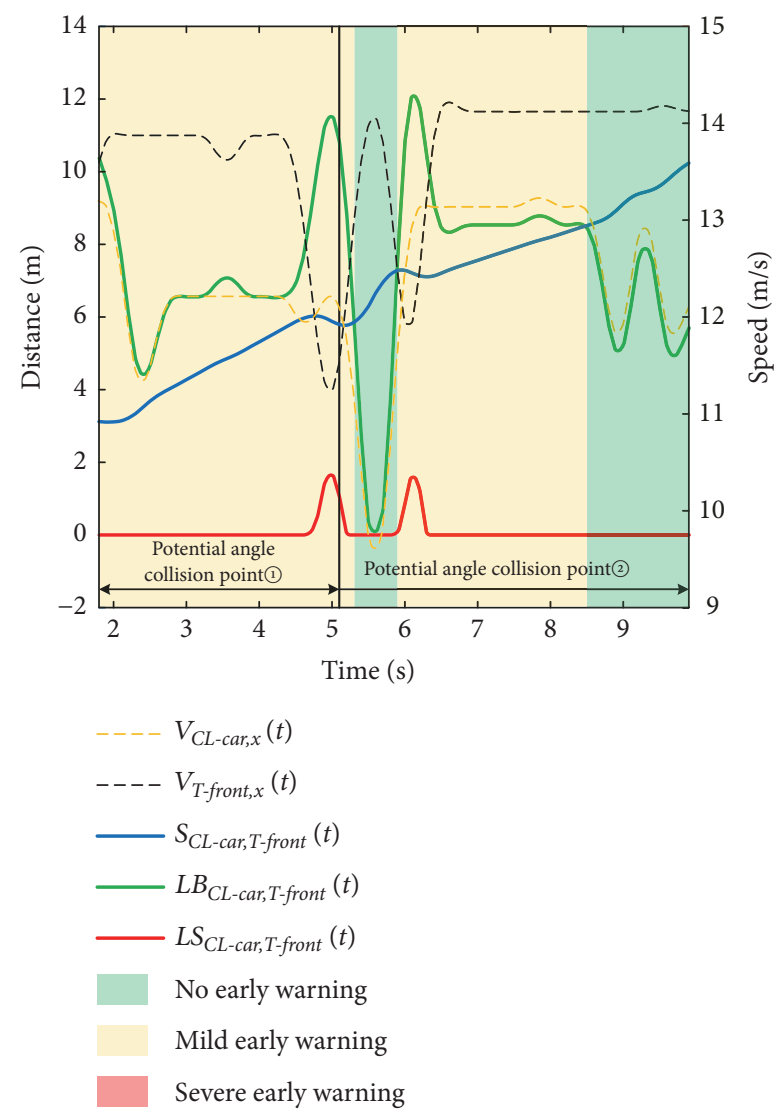

FIGURE 23: Distance and speed change between CL-car and T-front.

the sharp decline in the later period. The speed difference between the two vehicles gradually increases; the actual distance $S_{P \text {-back,CL-car }}(t)$ between the two vehicles increases; $L B_{P \text {-back,CL-car }}(t)$ is in the decrease trend; $L S_{P \text {-back,CL-car }}(t)$ is zero and remains unchanged. When $S_{P-b a c k, C L-c a r}(t)$ is greater than $L B_{P-b a c k, C L-c a r}(t)$, which satisfies the safety conditions, the early warning for P-back ends.

\subsubsection{Early Warning Analysis of CL-car and T-front.} Figure 23 shows in the early and middle stage of potential angle collision point (1) that since $v_{T-\text { front }, x}(t)$ is always larger than $v_{C L-c a r, x}(t), S_{C L-c a r, T-\text { front }}(t)$ increases, and $L S_{C L-c a r, T-f r o n t}(t)$ is zero and remains unchanged. Because $S_{C L \text {-car, } T \text {-front }}(t)$ is less than the minimum safety distance $L B_{C L \text {-car,T-front }}(t)$ and greater than $L S_{C L \text {-car,T-front }}(t), C L$-car should be given the mild early warning and pay attention to the emergency brake of preceding vehicle with appropriate deceleration.

In the late stage of the potential angle collision point (1), $v_{T \text {-front }, x}(t)$ decreases sharply with a large deceleration. Then $S_{C L-c a r, T-\text { front }}(t)$ becomes small, and $L B_{C L \text {-car }, T \text {-front }}(t)$ and $L S_{C L-c a r, T-f r o n t}(t)$ become large, reflecting the sensitivity and effectiveness of the minimum safety distance model in the work. Since $S_{C L-c a r, T-\text { front }}(t)$ is always greater than $L S_{C L \text {-car,T-front }}(t)$ and less than $L B_{C L \text {-car,T-front }}(t), C L$-car should be given the mild early warning at this stage and

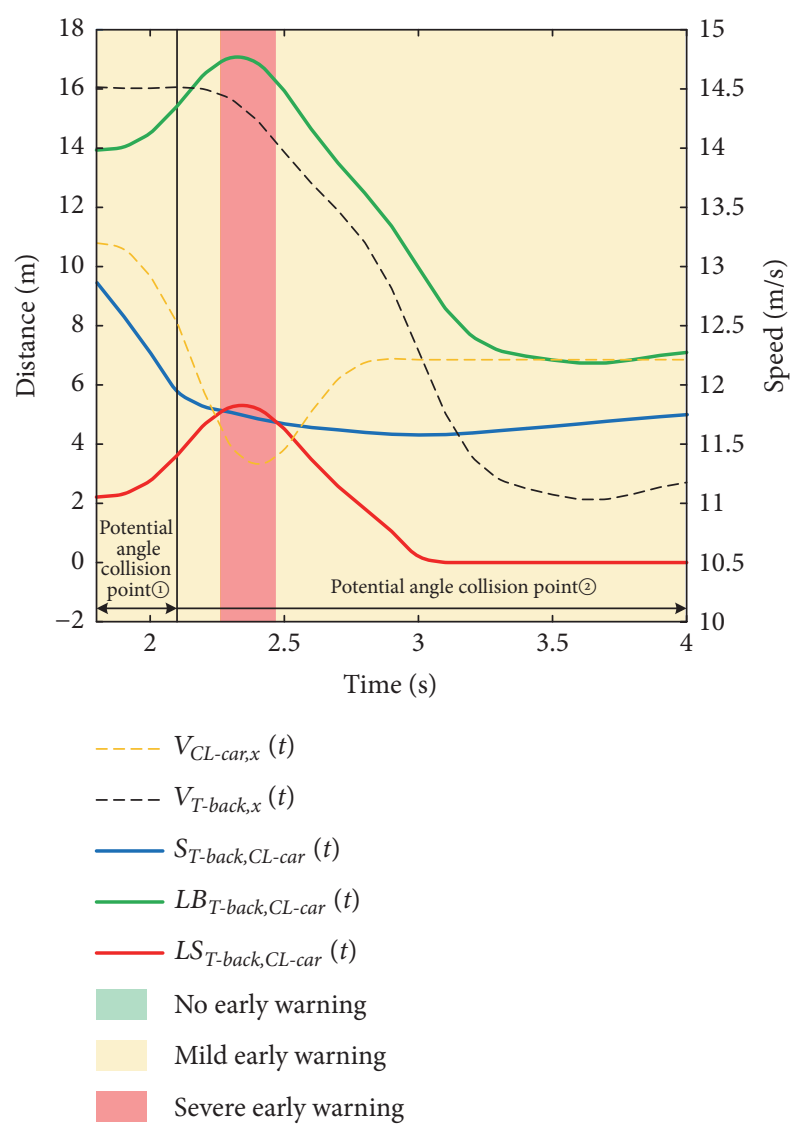

FIGURE 24: Distance and speed change between T-back and CL-car.

pay attention to the emergency braking of preceding with appropriate deceleration.

In the early stage of the potential angle collision (2), for the sudden decrease of $v_{T \text {-front } x}(t)$ in the later period of potential angle collision point (1), $C L$-car reacts rapidly and then decelerates, and $v_{T-\text { front, } x}(t)$ increases with a larger acceleration and exceeds $v_{C L-c a r, x}(t)$. It makes $S_{C L-c a r, T-\text { front }}(t)$ increase, $L B_{C L-c a r, T-\text { front }}(t)$ decrease, and $L S_{C L-c a r, T-\text { front }}(t)$ become zero. Since $S_{C L-c a r, T-\text { front }}(t)$ is greater than $L B_{C L \text {-car, } T \text {-front }}(t)$, which meets safety conditions, $C L$-car needs no early warning. In the middle and late stages of the potential angle collision point(2), since $v_{T-\text { front } x}(t)$ is always larger than $v_{C L-c a r, x}(t)$, the early warning for the CL-car is changed from the mild early warning to no early warning.

3.3.4. Early Warning Analysis of CL-car and T-back. Figure 24 shows that CL-car appears as the preceding vehicle and the vehicle T-back as the rear vehicle. At the process of potential angle collision point (1), $v_{T-\text { back, } x}(t)$ is always greater than $v_{C L-c a r, x}(t)$, which decreases $S_{T \text {-back,CL-car }}(t)$ and increases $L S_{T-b a c k, C L-c a r}(t)$ and $L B_{T \text {-back,CL-car }}(t)$. Since $S_{T-b a c k, C L-c a r}(t)$ is always greater than $L S_{T-b a c k, C L-c a r}(t)$ and less than $L B_{T-b a c k, C L-c a r}(t)$. T-back should be given the mild early warning and pay attention to the emergency brake of the rear vehicle with appropriate deceleration. 
In the early stage of the potential angle collision (2), $v_{T-b a c k, x}(t)$ is still higher than $v_{C L \text {-car }, x}(t)$. It makes $S_{\text {T-back,CL-car }}(t)$ even less than $L S_{T-b a c k, C L-c a r}(t)$. T-back should be given the severe early warning and decelerate immediately for safety.

In the middle and late stage of the potential angle collision point (2), for the sudden decrease of $v_{C L-c a r, x}(t)$ in the early stage, T-back perceives and then decelerates, which makes $v_{T-b a c k, x}(t)$ less than $v_{C L-c a r, x}(t)$ and $L S_{T-\text { back, } C L-c a r}(t)$ become zero. Then the two cars drive at a relatively stable speed difference, with the little change of $S_{T-b a c k, C L-c a r}(t)$ and $L B_{T-b a c k, C L-c a r}(t)$. Since $S_{T-b a c k, C L-c a r}(t)$ is greater than $L S_{T-b a c k, C L-c a r}(t)$ and less than $L B_{T-b a c k, C L-c a r}(t)$, T-back should be given mild early warning and pay attention to the front emergency brake with appropriate deceleration.

\section{Conclusions and Outlook}

The innovation of this paper is that we analyzed more comprehensive lane change scenes, and, based on this, position functions of potential angle collision points on the sides, front, and rear of lane change vehicles and obstacle vehicles were studied in this paper. At the same time, different early warning rules were formulated according to the relationship between the actual distance and the two kinds of minimum safety distances.

Under assumptions, this paper can use the constructed models to judge the potential collision risk of different degrees in the process of changing lanes in a timely and effective manner. The driver's behavior can be guided in real time according to early warning rules. Models and early warning rules established in this research could be applied to smart cars equipped with IOT (Internet of Things). The parameters such as speed and acceleration required by models were obtained through data acquisition and data interaction. Then, through the calculation and analysis of vehicle intelligent system, drivers were assisted to complete the safe lane change. The research content of this paper had good application value and practical significance for the development of advanced vehicle active safety system and the reduction of traffic accidents caused by lane change.

Models in this paper are mainly applicable to the analysis and early warning of lane change in long straight sections and longitudinal slope sections. Therefore, in the followup research, it is necessary to analyze the flat and curved sections. At the same time, the follow-up research will consider the influence of driver's psychological role and the external environment on the minimum safe distance model and then modify the model.

\section{Data Availability}

The data for the five vehicles used in the work comes from the video data of vehicles travelling on Interstate 80 in Emeryville, California, collected by Berkeley Highway Laboratory (accessibility of data acquisition: ops.fhwa.dot.gov/trafficanalysistools/ngsim.htm).

\section{Conflicts of Interest}

The authors declare that there are no conflicts of interest regarding the publication of this article.

\section{Acknowledgments}

The work was funded by the National Natural Science Foundation of China (Grant no. 51308249).

\section{References}

[1] G. Gu, Cooperative Lane-changing Trajectory Planning ofIntelligent Vehicles with Consideration of DriverCharacteristics, South China University of Technology, 2008.

[2] H. Jula, E. B. Kosmatopoulos, and P. A. Ioannou, "Collision avoidance analysis for lane changing and merging," IEEE Transactions on Vehicular Technology, vol. 49, no. 6, pp. 22952308, 2000.

[3] J. A. Laval and C. F. Daganzo, "Lane-changing in traffic streams," Transportation Research Part B: Methodological, vol. 40, no. 3, pp. 251-264, 2006.

[4] H. Wan, Z. Liu, and H. Wang, "Rear-end and lane-changing collisions avoidance theoretical models of the multi-lane freeway in disaster weather," Procedia - Social and Behavioral Sciences, vol. 96, pp. 70-81, 2013.

[5] C. Hatipoglu, U. Ozguner, and K. Redmill, "Automated lane change controller design," IEEE Transactions on Intelligent Transportation Systems, vol. 4, no. 1, pp. 13-22, 2003.

[6] A. Vahidi and A. Eskandarian, "Research advances in intelligent collision avoidance and adaptive cruise control," IEEE Transactions on Intelligent Transportation Systems, vol. 4, no. 3, pp. 143153, 2003.

[7] Z. Shiller and S. Sundar, "Emergency lane-change maneuvers of autonomous vehicles," Journal of Dynamic Systems, Measurement, and Control, vol. 120, no. 1, pp. 37-44, 1998.

[8] C. L. Wang, Research on Highway Anti-Collision Alarm System Based on Zigbee, Harbin Institute of Technology, 2010.

[9] C. Wang, Research on Several Key Problems of Vehicle Lane Change Warning, Chang'an University, 2012.

[10] J. Feng and J. Ruan, "Study on intelligent vehicle lane change path planning and control simulation," in Proceedings of the 2006 IEEE International Conference on Information Acquisition, ICIA 2006, pp. 683-688, China, August 2006.

[11] G. Xu, L. Liu, Y. Ou, and Z. Song, "Dynamic modeling of driver control strategy of lane-change behavior and trajectory planning for collision prediction," IEEE Transactions on Intelligent Transportation Systems, vol. 13, no. 3, pp. 1138-1155, 2012.

[12] Y. Luo, Y. Xiang, K. Cao, and K. Li, "A dynamic automated lane change maneuver based on vehicle-to-vehicle communication," Transportation Research Part C: Emerging Technologies, vol. 62, pp. 87-102, 2015.

[13] H. Z. Zhu, S. Chang, W. Zhang, F. Wu, and L. Lu, "Online vehicle front-rear distance estimation with urban contextaware trajectories," IEEE Transactions on Vehicular Technology, vol. 67, no. 2, pp. 1063-1074, 2018.

[14] Y. Zhu and J. Chen, "Research on the minimum safe distance of vehicle lane changing based on fish swarm grouping behavior," Software Guide, vol. 17, no. 3, pp. 23-27, 2018.

[15] C. Olaverri-Monreal, G. C. Krizek, F. Michaeler, R. Lorenz, and M. Pichler, "Collaborative approach for a safe driving distance 
using stereoscopic image processing," Future Generation Computer Systems, vol. 95, pp. 880-889, 2019.

[16] Q. Luo and L. H. Xu, "Car-following model based on the minimum safety distance and simulation study," Science Technology and Engineering, vol. 10, no. 2, pp. 569-573, 2010.

[17] S. M. Wang, J. Y. Xu, Q. Luo et al., "A safety warning model for lane changing on highway," Journal of South China University of Technology (Natural Science Edition), vol. 42, no. 12, pp. 40-50, 2014.

[18] R. S. Tomar, S. Verma, R. S. Kushwah, and G. S. Tomar, "Collision avoidance warning for safe lane change," in Proceedings of the 2013 International Conference on Communication Systems and Network Technologies (CSNT 2013), vol. 12, pp. 385-389, April 2013.

[19] Y. Luo, Y. Xiang, K. Cao, and K. Li, "A dynamic automated lane change maneuver based on vehicle-to-vehicle communication," Transportation Research Part C: Emerging Technologies, vol. 62, pp. 87-102, 2016.

[20] A. Kanaris, E. B. Kosmatopoulos, P. A. Loannou et al., "Strategies and spacing requirements for lane changing and merging in automated highway systems," IEEE Transactions on Vehicular Technology, vol. 50, no. 6, pp. 1568-1581, 2001.

[21] X. Q. Yin, P. J. Liu, X. D. Zou et al., "Optimization and simulation of the minimum safety distance of car following model," Information Technology Journal, vol. 12, no. 17, pp. 41014105, 2013.

[22] Y. H. Wu, J. X. Xie, L. H. Du, and Z. Hou, "Analysis on traffic safety distance of considering the deceleration of the current vehicle," in Proceedings of the 2009 Second International Conference on Intelligent Computation Technology and Automation, pp. 491-494, Changsha, China, October 2009.

[23] H. Yuan, G. Shi, X. Huang, and J. Cheng, "Braking model of stopping sight distance," Journal of Southeast University (Natural Science Edition), vol. 39, no. 4, pp. 859-862, 2009.

[24] R. H. Zhang, Z. C. He, H. W. Wang, F. You, and K. Li, "Study on self-tuning tyre friction control for developing main-servo loop integrated chassis control system," IEEE Access, vol. 5, pp. 6649-6660, 2017.

[25] X. J. Sun, H. Zhang, W. J. Meng, R. Zhang, K. Li, and T. Peng, "Primary resonance analysis and vibration suppression for the harmonically excited nonlinear suspension system using a pair of symmetric viscoelastic buffers," Nonlinear Dynamics, vol. 94, no. 2, pp. 1243-1265, 2018.

[26] B. Park, Y. Chen, and J. Hourdos, "Opportunities for preventing rear-end crashes: Findings from the analysis of actual freeway crash data," Journal of Transportation Safety \& Security, vol. 3, no. 2, pp. 95-107, 2011.

[27] N. H. Jiang and J. G. Yu, "Study on rear-end avoidance model of highway based on RBF neural network," Forest Engineering, vol. 26, no. 5, pp. 60-65, 2010.

[28] B. E. Heinrichs, B. D. Allin, J. J. Bowler, and G. P. Siegmund, "Vehicle speed affects both pre-skid braking kinematics and average tire/roadway friction," Accident Analysis \& Prevention, vol. 36, no. 5, pp. 829-840, 2004.

[29] H. Y. Xiong, X. L. Zhu, and R. H. Zhang, "Energy recovery strategy numerical simulation for dual axle drive pure electric vehicle based on motor loss model and big data calculation," Complexity, vol. 2018, Article ID 4071743, 14 pages, 2018.

[30] J. Rehkopf, Automobile Brake Systems, Pearson Prentice Hall, Upper Saddle River, NJ, USA, 2008.
[31] J. Y. Lian and X. Y. Hua, "Study of mathematics model for rear end collision avoidance system," China Journal of Highway and Transport, vol. 18, no. 3, pp. 123-126, 2005.

[32] H. Wang, S. Zhang, W. Quan, and X. Liu, "Study on safety distance model of fleet based on vehicle communication," Procedia - Social and Behavioral Sciences, vol. 96, no. 6, pp. 2698-2705, 2013. 


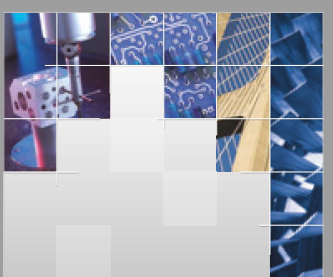

\section{Enfincering}
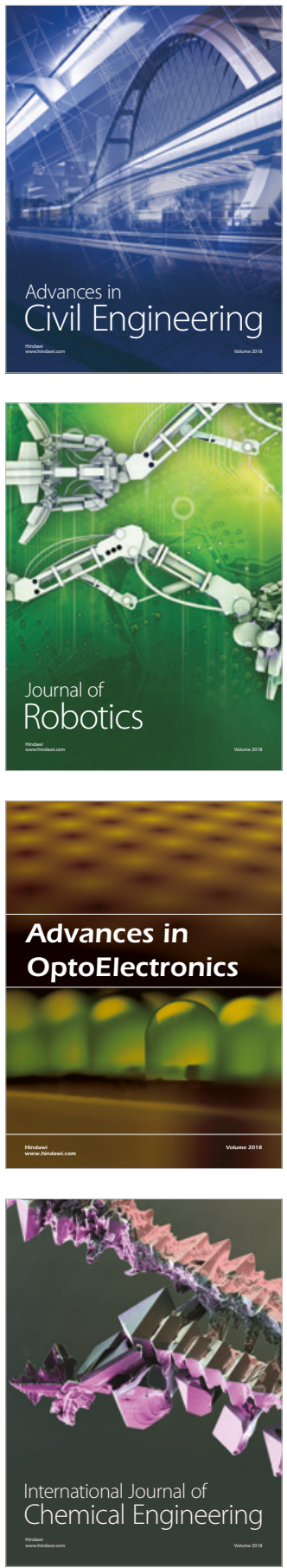

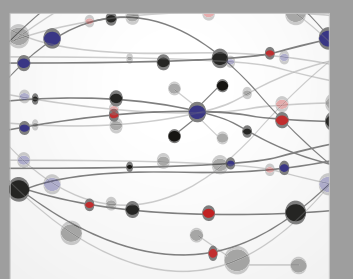

\section{Rotating \\ Machinery}

The Scientific World Journal

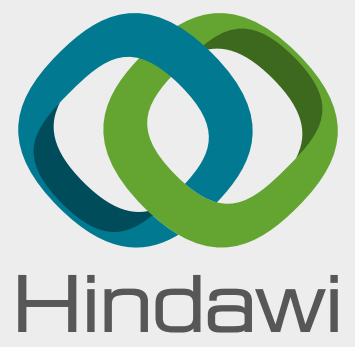

Submit your manuscripts at

www.hindawi.com
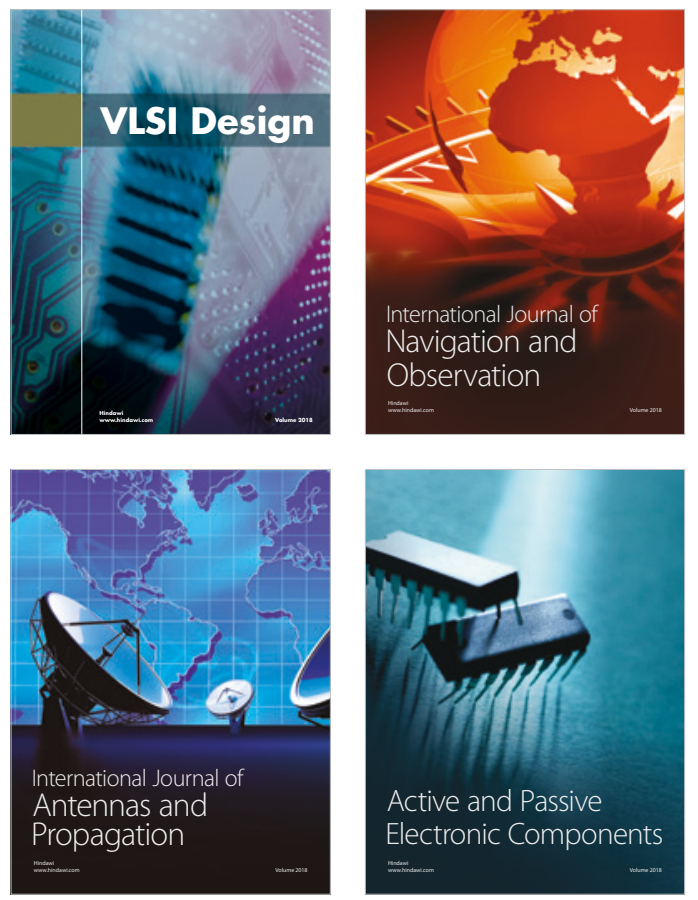
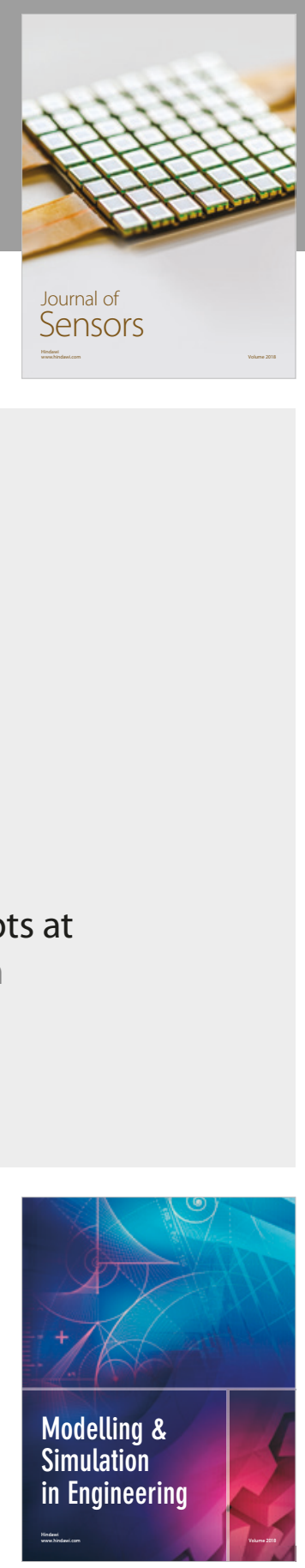

\section{Advances \\ Multimedia}
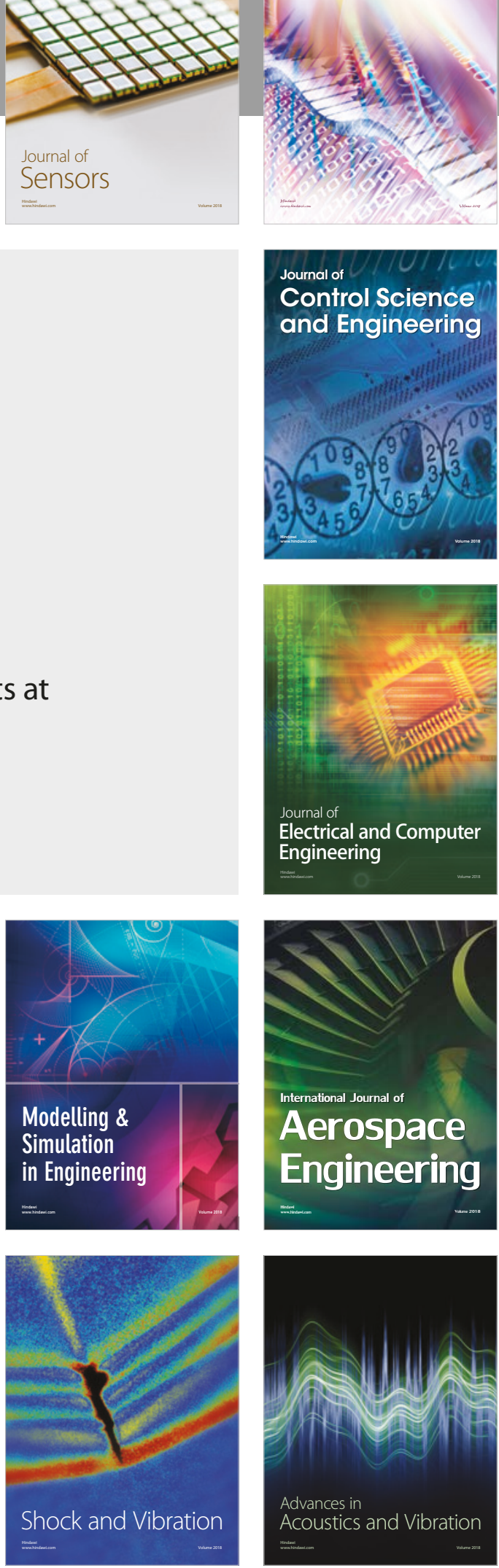